\title{
A Novel Technique for Varus Tibial Cutting for Oxford Unicompartmental Knee Arthroplasty
}

\author{
Takafumi Hiranaka, MD, Toshikazu Tanaka, MD, Takaaki Fujishiro, MD, Kenjiro Okimura, MD, \\ Rika Shigemoto, MD, Shotaro Araki, MD, Ryo Okada, MD, Ryohei Nako, MD, Koji Okamoto, MD \\ Department of Orthopaedic Surgery and Joint Surgery Centre, Takatsuki General Hospital, Takatsuki, Japan
}

To reduce the stress on the medial tibial cortex and to decrease the risk of fracture, a varus cut of the tibia appears to be a reasonable alternative to the orthogonal cut by conventional methods. We present a new instrument and procedure, which enables a varus tibial cut for Oxford unicompartmental knee arthroplasty. We used a custom-made, slidable fixator instead of the standard fixator to set the extramedullary rod on the leg. We also made a numeric formula and a chart to arrange the varus cutting angle using the length of the mediolateral shift of the distal end and the longitudinal extension length of the extramedullary tibial rod. A varus cut up to $4.5^{\circ}$ can be controlled. This technique is a simple and useful means of obtaining a varus tibial cut for Oxford unicompartmental knee arthroplasty.

Keywords: Arthroplasty, Replacement, Knee, Operation, Alignment

The Oxford unicompartmental knee arthroplasty (OUKA) is characterized by mobile meniscal bearing, which enables reduction of polyethene wear and retention of ligament balance. ${ }^{1)}$ Proper component alignments are essential for desired clinical outcomes, and the improved instrumentation set, the Microplasty, has been reported to achieve this reliably. ${ }^{2)}$ Studies have shown that OUKA can acquire good clinical outcomes, but a higher risk of tibial fracture after OUKA has been reported, especially in Asian countries. A recent study revealed that such a high frequency of complication could be caused by tibial morphology, such as a very overhanging medial condyle found in a proximal tibia vara, rather than by technical errors. ${ }^{3)}$ A slight varus cut has been recommended to prevent fractures because it can distance the keel from the cortex. Regarding fixed-bearing UKA, a slight varus implantation has been a popular technique to reduce mechanical stress ${ }^{4)}$ and cutting instru-

Received July 30, 2020; Accepted September 14, 2020

Correspondence to: Takafumi Hiranaka, MD

Department of Orthopaedic Surgery and Joint Surgery Centre, Takatsuki General Hospital, 1-3-13 Kosobe-Cho, Takatsuki City, Osaka Prefecture, 5691115 Japan

Tel: +81-726813801, Fax: +81-726823834

E-mail: takafumi.hiranaka@gmail.com ments, and computer navigation and robotic surgery can be used to adjust the varus cut angle. In OUKA, however, only orthogonal cuts against the tibial axis are available. We, therefore, devised a custom-made, slidable fixator to set the extramedullary rod on the leg and used it instead of the standard fixator provided by the manufacturer. We describe our custom-made cutting guide and demonstrate how to use it to adjust the varus cut angle.

\section{TECHNIQUE}

We used a custom-made slidable fixator (Fig. 1). It can be used for the fixation of the tibial rod to the distal tibia using the standard rubber straps. The fixator can be connected to the distal part of the tibial rod, and similar to the standard fixator, it can be used to adjust the sagittal alignment of the rod. A mediolateral adjustment can be made up to $2 \mathrm{~cm}$ on both sides, and the amount of the slide is visible on the ruler.

The amount of the varus angle can be calculated using mediolateral displacement (MLD) and the extension length (EPL). The EPL is measured by a ruler as the length of the flat surface of the rod (Fig. 1). The pivot of the tibial rod rotation is the most lateral hole for the tibial cutting block fixation, so the varus angle is geometrically calculat- 
ed using the MLD and the total rod length (distance from the lateral hole to the fixator) (Fig. 2). Given the EPL $=0$, the rod length is $25.7 \mathrm{~cm}$; we used the following formula:

$$
\text { MLD cm }=\text { Tan }(\text { varus angle }) \times(25.7 \mathrm{~cm}+\mathrm{EPL} \mathrm{cm})
$$

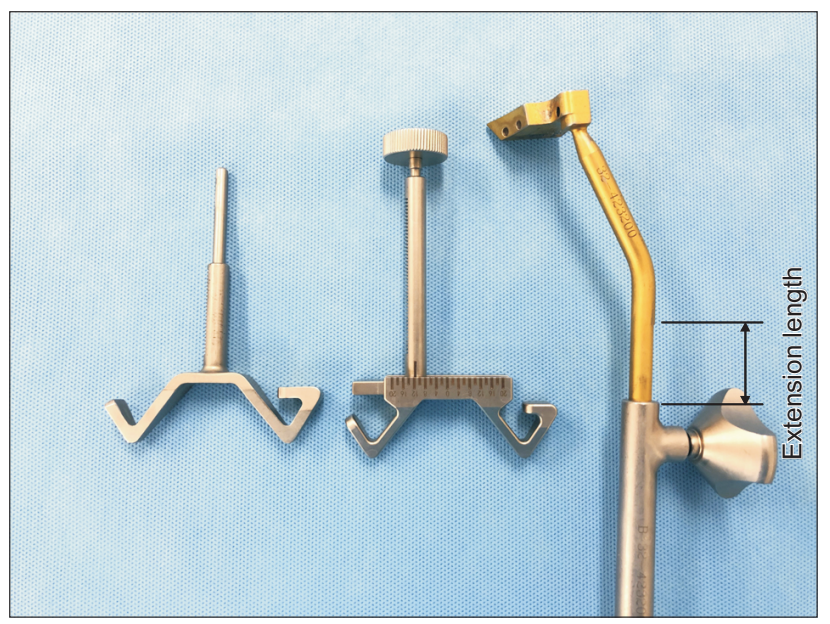

Fig. 1. Extramedullary tibial alignment rods for the Oxford unicompartmental knee arthroplasty: the conventional fixator tool (left) and the custom-made, slidable fixator tool (center). The distal end of the extramedullary rod is connected with the leg using the fixator, and the degree of varus angulation can be adjusted referring to the scale on the fixator and the length of the extension of the extramedullary rod. The extension length of the rod is measured (arrow), and the varus angle is calculated using the chart or the formula.
For this calculation, a Microsoft Excel spreadsheet (Supplementary Material 1) and a summarized chart (Table 1) are provided.

\section{Radiographic Assessment}

We evaluated tibial component angle against the tibial axis (Fig. 3) in 28 knees in 20 consecutive patients who underwent OUKA using a conventional instrument from November to December in 2019 (conventional group) and

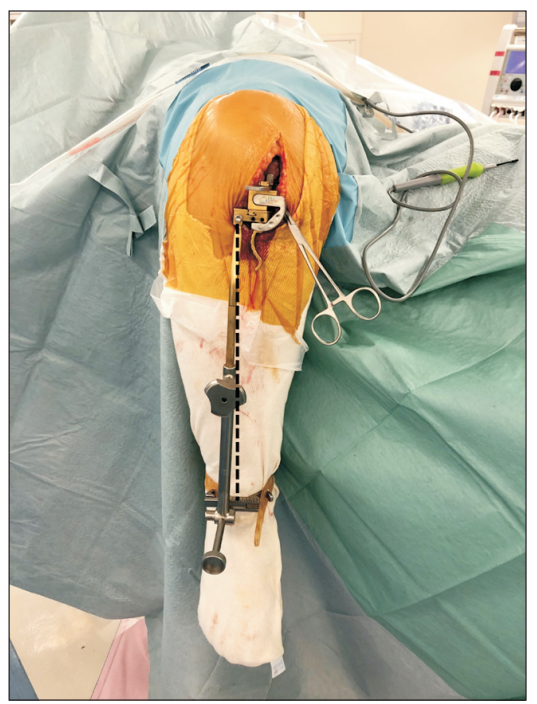

Fig. 2. A varus setting of the extramedullary rod. The rod slants varus against the tibial axis (dotted line).

Table 1. Length of the Lateral Shift of the Fixator Corresponding with the Longitudinal Extension of the Extramedullary Rod and the Varus Angle

\begin{tabular}{|c|c|c|c|c|c|c|c|c|c|}
\hline \multirow{2}{*}{$\begin{array}{l}\text { Extension } \\
\text { length }\end{array}$} & \multicolumn{9}{|c|}{ Varus angle $\left({ }^{\circ}\right)$} \\
\hline & 0.5 & 1.0 & 1.5 & 2.0 & 2.5 & 3.0 & 3.5 & 4.0 & 4.5 \\
\hline $0 \mathrm{~cm}$ & $0.2 \mathrm{~cm}$ & $0.4 \mathrm{~cm}$ & $0.7 \mathrm{~cm}$ & $0.9 \mathrm{~cm}$ & $1.1 \mathrm{~cm}$ & $1.3 \mathrm{~cm}$ & $1.6 \mathrm{~cm}$ & $1.8 \mathrm{~cm}$ & $2.0 \mathrm{~cm}$ \\
\hline $1 \mathrm{~cm}$ & $0.2 \mathrm{~cm}$ & $0.5 \mathrm{~cm}$ & $0.7 \mathrm{~cm}$ & $0.9 \mathrm{~cm}$ & $1.2 \mathrm{~cm}$ & $1.4 \mathrm{~cm}$ & $1.6 \mathrm{~cm}$ & $1.9 \mathrm{~cm}$ & $2.1 \mathrm{~cm}$ \\
\hline $2 \mathrm{~cm}$ & $0.2 \mathrm{~cm}$ & $0.5 \mathrm{~cm}$ & $0.7 \mathrm{~cm}$ & $1.0 \mathrm{~cm}$ & $1.2 \mathrm{~cm}$ & $1.5 \mathrm{~cm}$ & $1.7 \mathrm{~cm}$ & $0.9 \mathrm{~cm}$ & $2.2 \mathrm{~cm}$ \\
\hline $3 \mathrm{~cm}$ & $0.3 \mathrm{~cm}$ & $0.5 \mathrm{~cm}$ & $0.8 \mathrm{~cm}$ & $1.0 \mathrm{~cm}$ & $1.3 \mathrm{~cm}$ & $1.5 \mathrm{~cm}$ & $1.8 \mathrm{~cm}$ & $2.0 \mathrm{~cm}$ & \\
\hline $4 \mathrm{~cm}$ & $0.3 \mathrm{~cm}$ & $0.5 \mathrm{~cm}$ & $0.8 \mathrm{~cm}$ & $1.0 \mathrm{~cm}$ & $1.3 \mathrm{~cm}$ & $1.6 \mathrm{~cm}$ & $1.8 \mathrm{~cm}$ & $2.1 \mathrm{~cm}$ & \\
\hline $5 \mathrm{~cm}$ & $0.3 \mathrm{~cm}$ & $0.5 \mathrm{~cm}$ & $0.8 \mathrm{~cm}$ & $1.1 \mathrm{~cm}$ & $1.3 \mathrm{~cm}$ & $1.6 \mathrm{~cm}$ & $1.9 \mathrm{~cm}$ & $2.1 \mathrm{~cm}$ & \\
\hline $6 \mathrm{~cm}$ & $0.3 \mathrm{~cm}$ & $0.6 \mathrm{~cm}$ & $0.8 \mathrm{~cm}$ & $1.1 \mathrm{~cm}$ & $1.4 \mathrm{~cm}$ & $1.7 \mathrm{~cm}$ & $1.9 \mathrm{~cm}$ & $2.2 \mathrm{~cm}$ & \\
\hline $7 \mathrm{~cm}$ & $0.3 \mathrm{~cm}$ & $0.6 \mathrm{~cm}$ & $0.9 \mathrm{~cm}$ & $1.1 \mathrm{~cm}$ & $1.4 \mathrm{~cm}$ & $1.7 \mathrm{~cm}$ & $2.0 \mathrm{~cm}$ & $2.3 \mathrm{~cm}$ & \\
\hline $8 \mathrm{~cm}$ & $0.3 \mathrm{~cm}$ & $0.6 \mathrm{~cm}$ & $0.9 \mathrm{~cm}$ & $1.2 \mathrm{~cm}$ & $1.5 \mathrm{~cm}$ & $1.8 \mathrm{~cm}$ & $2.1 \mathrm{~cm}$ & & \\
\hline $9 \mathrm{~cm}$ & $0.3 \mathrm{~cm}$ & $0.6 \mathrm{~cm}$ & $0.9 \mathrm{~cm}$ & $1.2 \mathrm{~cm}$ & $1.5 \mathrm{~cm}$ & $1.8 \mathrm{~cm}$ & $2.1 \mathrm{~cm}$ & & \\
\hline $10 \mathrm{~cm}$ & $0.3 \mathrm{~cm}$ & $0.6 \mathrm{~cm}$ & $0.9 \mathrm{~cm}$ & $1.2 \mathrm{~cm}$ & $1.6 \mathrm{~cm}$ & $1.9 \mathrm{~cm}$ & $2.2 \mathrm{~cm}$ & & \\
\hline
\end{tabular}




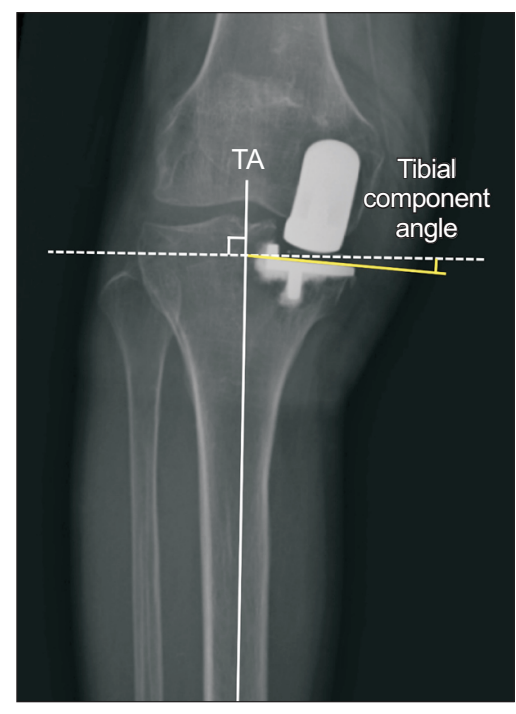

Fig. 3. Radiographic measurement. Tibial axis (TA): the line between the ankle center and the midpoint of the intercondylar eminences. The tibial component angle was defined as the angle between the line perpendicular to the TA (dotted line) and the line parallel to the tibial component surface (yellow line) on the postoperative anteroposterior radiograph. A positive value indicates the varus alignment of the tibial component.

in 32 knees in 20 consecutive patients who underwent OUKA using the new varus cut instrument (varus cutting group). The average component angle was $0.16^{\circ} \pm 1.14^{\circ}$ varus for the conventional group and $3.19^{\circ} \pm 1.32^{\circ}$ varus for the varus cutting group. The mean difference was $3.03^{\circ}$, and the difference was significant $(p<0.0001)$ with a large effect size (Cohen's $d=2.37$ ). A power analysis was performed using $\mathrm{EZR}^{5)}$ running on $\mathrm{R}$, which showed that the required sample size was 7 in each group, indicating the sample size was sufficient.

\section{DISCUSSION}

Our technique aims to enable an intentional varus tibial cut at the desired angle in OUKA by using an additional simple optional modification. The tibial cutting guide has been used continuously from the Phase- 3 to the Microplasty instrumentation versions of OUKA. Although a very stable tibial horizontal cut has been achieved, ${ }^{2)}$ it has only been possible to make orthogonal horizontal cuts against the tibial axis. This is the first report of a technique that enables an intentional varus cut in OUKA.

The varus cut angle can be set to $4.5^{\circ}$ and $3.0^{\circ}$ when the EPL is minimum $(0 \mathrm{~cm})$ and maximum $(10 \mathrm{~cm})$, respectively. Studies have shown that a slight varus cut is beneficial to stress distribution. Inoue et al. ${ }^{6)}$ suggested a $6^{\circ}$ of varus cut was the best to avoid the stress concentra- tion to the medial cortex of the medial tibia. They also recommended the $3^{\circ}$ varus cut, however, because in some reports, an excessive varus cut was shown to decrease the implant survival in UKA. ${ }^{4)}$ Another computer simulation study recommended a slight varus implantation to reduce the peak stress on the medial cortex in the mobile-bearing OUKA. ${ }^{7)}$ Sekiguchi et al. ${ }^{8)}$ reported a computer simulation study where a $2^{\circ}$ varus cut showed the best kinematics and ligament tension. An intended varus cut up to $3^{\circ}$ was indicated by these results to be both beneficial and practical. Our technique enables medially sloped cuts by adding a simple part to the instrument, and it can also follow the natural medial slope of the medial tibial plateau. A spoon gauge is used to decide the femoral component size and to measure the gap between the femoral and tibial articular surfaces. The coronal alignment of the spoon gauge follows the medial slope of the tibial plateau, which faces the flat undersurface of the gauge. However, the medial inclination is corrected to be orthogonal to the tibial rod. The gap can eventually expand, however, resulting in a higher tibial cut and a narrower flexion gap. Conversely, a medial sloped cut would retain the desired gap.

Varus implantation can also be beneficial for load transmission because the joint line can be made parallel to the floor. ${ }^{4)}$ Sampath et al. ${ }^{9)}$ showed that the trabecular orientation could be changed according to the leg alignment and direction of the load across the joint. Implantation of the tibial tray in the same orientation might be best supported by the underlying trabecular.

Another solution for the varus tibial cut might be the medial displacement of the cutting block. Our chart also provides information on how much the block should be moved medially to acquire the desired varus angle. To get a $3^{\circ}$ varus cut, a minimum of $1.3 \mathrm{~cm}$ up to $2.0 \mathrm{~cm}$ of medial displacement is necessary. In this situation, the fixation pin for the cutting block penetrates the cortex adjacent to the keel slit, resulting in an increased risk of fracture. Brumby et al. ${ }^{10)}$ reported that an improperly made pinhole could be a risk of fracture. An advantage of our method is that the pinhole is made at the center of the tibia without danger of such fractures.

We are aware of some limitations concerning the technique. Firstly, this is possible by the use of the unique slidable fixator tool. We have used a custom-made instrument, but it will enter manufacture shortly. Secondly, no available reports have shown better clinical outcomes after varus implantation than by an orthogonal cut after the OUKA. Riviere et al. ${ }^{11)}$ reported on the varus tibial cut. Although it was beneficial in terms of tibial component fitting, long-term clinical outcome was not shown. Further 
Hiranaka et al. Tibial Varus Cut in Unicompartmental Knee Arthroplasty

Clinics in Orthopedic Surgery • Vol. 12, No. 4, $2020 \bullet$ www.ecios.org

research would be needed to prove the benefit of the varus cut in the OUKA. Despite the limitations, the new fixator tool enables intentional varus tibial cuts. A further clinical study is required.

\section{CONFLICT OF INTEREST}

No potential conflict of interest relevant to this article was reported.

\section{SUPPLEMENTARY MATERIAL}

Supplementary material is available in the electronic version of this paper at the CiOS website, www.ecios.org.

\section{REFERENCES}

1. Pietschmann MF, Ficklscherer A, Wohlleb L, Schmidutz F, Jansson V, Muller PE. UKA can be safely revised to primary knee arthroplasty by using an autologous bone plate from the proximal lateral tibia. J Arthroplasty. 2014;29(10):19915.

2. Walker T, Heinemann P, Bruckner T, Streit MR, Kinkel S, Gotterbarm T. The influence of different sets of surgical instrumentation in Oxford UKA on bearing size and component position. Arch Orthop Trauma Surg. 2017;137(7):895902.

3. Hiranaka T, Yoshikawa R, Yoshida K, et al. Tibial shape and size predicts the risk of tibial plateau fracture after cementless unicompartmental knee arthroplasty in Japanese patients. Bone Joint J. 2020;102(7):861-7.

4. Asada S, Inoue S, Tsukamoto I, Mori S, Akagi M. Obliquity of tibial component after unicompartmental knee arthroplasty. Knee. 2019;26(2):410-5.

5. Kanda Y. Investigation of the freely available easy-to-use software 'EZR' for medical statistics. Bone Marrow Transplant. 2013;48(3):452-8.

6. Inoue S, Akagi M, Asada S, Mori S, Zaima H, Hashida M. The Valgus inclination of the tibial component increases the risk of medial tibial condylar fractures in unicompartmental knee arthroplasty. J Arthroplasty. 2016;31(9):2025-30.

7. Chatellard R, Sauleau V, Colmar M, et al. Medial unicompartmental knee arthroplasty: does tibial component position influence clinical outcomes and arthroplasty survival? Orthop Traumatol Surg Res. 2013;99(4 Suppl):S219-25.

8. Sekiguchi K, Nakamura S, Kuriyama S, et al. Effect of tibial component alignment on knee kinematics and ligament tension in medial unicompartmental knee arthroplasty. Bone Joint Res. 2019;8(3):126-35.

9. Sampath SA, Lewis S, Fosco M, Tigani D. Trabecular orientation in the human femur and tibia and the relationship with lower-limb alignment for patients with osteoarthritis of the knee. J Biomech. 2015;48(6):1214-8.

10. Brumby SA, Carrington R, Zayontz S, Reish T, Scott RD. Tibial plateau stress fracture: a complication of unicompartmental knee arthroplasty using 4 guide pinholes. J Arthroplasty. 2003;18(6):809-12.

11. Riviere C, Harman C, Leong A, Cobb J, Maillot C. Kinematic alignment technique for medial OXFORD UKA: an in-silico study. Orthop Traumatol Surg Res. 2019;105(1):6370. 